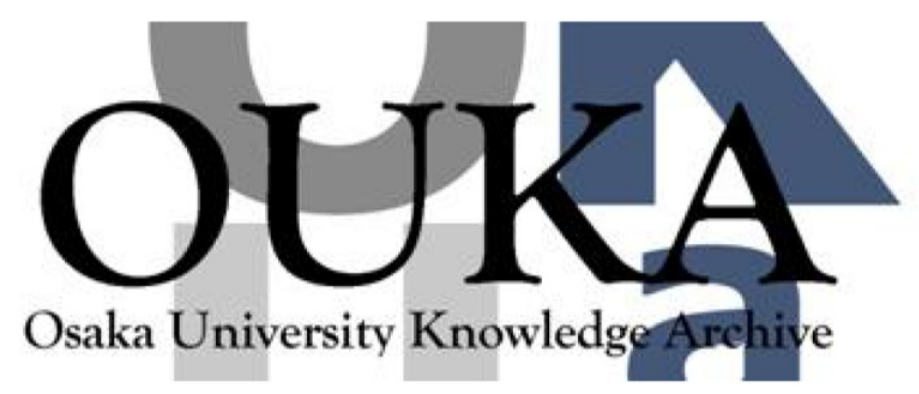

\begin{tabular}{|c|l|}
\hline Title & $\begin{array}{l}\text { Optomechanical effect in ferroelectric liquid } \\
\text { crystal freely suspended films }\end{array}$ \\
\hline Author(s) & $\begin{array}{l}\text { Yablonskii, Serguei V.; Nakano, Kazuyuki; } \\
\text { Ozaki, Masanori et al. }\end{array}$ \\
\hline Citation & Journal of Applied Physics. 94(8) p.5206-p.5209 \\
\hline Issue Date & $2003-09-30$ \\
\hline oaire:version & VoR \\
\hline URL & https://hdl. handle. net/11094/75849 \\
\hline rights & \\
\hline Note & \\
\hline
\end{tabular}

Osaka University Knowledge Archive : OUKA

https://ir. Library. osaka-u. ac. jp/

Osaka University 


\section{Optomechanical effect in ferroelectric liquid crystal freely suspended films}

Cite as: Journal of Applied Physics 94, 5206 (2003); https://doi.org/10.1063/1.1611637

Submitted: 07 April 2003 . Accepted: 29 July 2003 . Published Online: 30 September 2003

Serguei V. Yablonskii, Kazuyuki Nakano, Masanori Ozaki, Mikhail V. Kozlovsky, and Katsumi Yoshino

\section{ARTICLES YOU MAY BE INTERESTED IN}

Submicrosecond bistable electro-optic switching in liquid crystals

Applied Physics Letters 36, 899 (1980); https://doi.org/10.1063/1.91359

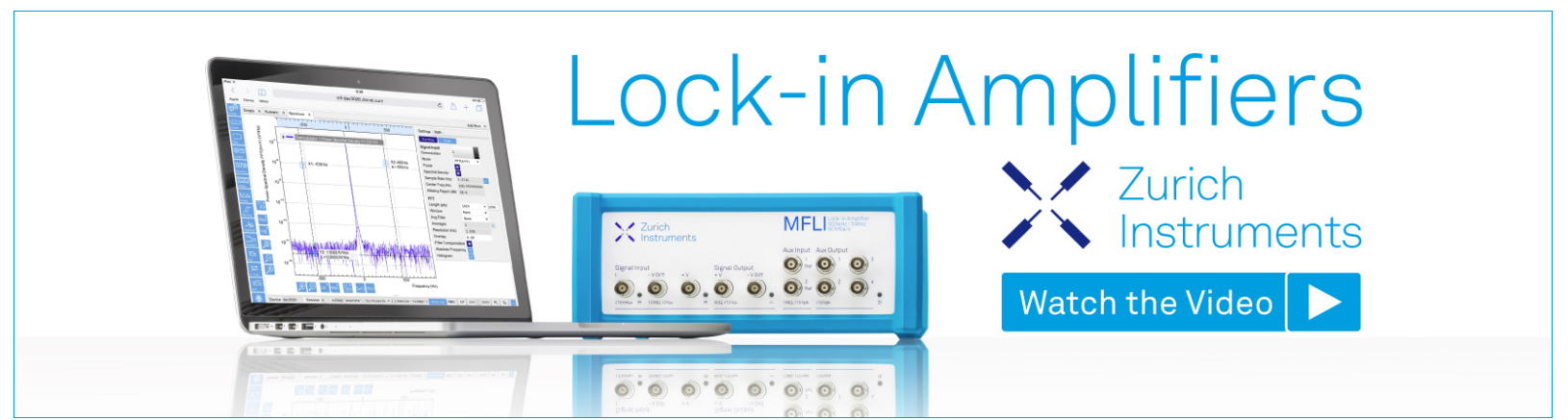




\title{
Optomechanical effect in ferroelectric liquid crystal freely suspended films
}

\author{
Serguei V. Yablonskii \\ Institute of Crystallography, Russian Academy of Science, 117333 Moscow, Russia
}

Kazuyuki Nakano and Masanori Ozaki

Department of Electronic Engineering, Graduate School of Engineering, Osaka University, 2-1 Yamada-oka, Suita, Osaka 565-0871, Japan

Mikhail V. Kozlovsky

Institut für Physikalische Chemie, TUD, Petersenstrasse 20, Darmstadt, 64287, Germany

Katsumi Yoshino a)

Department of Electronic Engineering, Graduate School of Engineering, Osaka University, 2-1 Yamada-oka, Suita, Osaka 565-0871, Japan

(Received 7 April 2003; accepted 29 July 2003)

Optomechanical effect in vibrating ferroelectric liquid crystal freely suspended films have been observed. In the experiment the liquid crystal films are doped with a photochromic dye which undergoes trans-cis isomerization. Photoisomerization induces the change both in a switching potential of the host liquid crystal and the film surface tension. We observed also a nonlinear dependence of photoinduced variation of the surface tension as function of the dye concentration. Such dependence strongly supports the possibility of amplification of photochemical effect in liquid crystals. The optomechanical effect might be exploited for the remote control of the sensors using freely suspended films, and more generally for the investigation of the cooperative phenomena in liquid crystals. (C) 2003 American Institute of Physics. [DOI: 10.1063/1.1611637]

\section{INTRODUCTION}

Unlike the standard case of a liquid crystal sample in a confined geometry, the surface of a freely suspended liquid crystal film can easily be deformed under the action of weak acoustic or electric fields. ${ }^{1-5}$ Such a film is considered as perfect membrane, whose vibrational motion depends only on the film geometry, isotropic surface tension and homogeneous two-dimensional density (including the inertia of the air moving with the film). ${ }^{6-9}$ Change in any of these parameters strongly influences resonant properties of the vibrating film, for example, the pressure gauge employing freely suspended film was shown to indicate the change of air pressure by of only 0.5 Torr. ${ }^{3,10}$ Here we present experimental evidence of so-called optomechanical effect ${ }^{11-14}$ in a freely suspended film made from a ferroelectric liquid crystal (denoted CS-1029) doped with a photoreactive dye (an azobenzene derivative, denoted $D^{* 55}$ ). In the experiment, we studied transverse mechanical oscillations of freely suspended films induced by lateral sinusoidal electric field, the effect being linear upon the field applied. Exposure of the vibrating film to UV and VIS light results in a reversible shift of resonant frequencies due to the photoisomerization process. In addition, we see the reversible changes of film vibration amplitudes (up to $100 \%$ ), which seem to be associated with the influence of cis-conformers on the magnitude of the spontaneous polarization of the ferroelectric film. ${ }^{15-18}$ We observed also a nonlinear dependence of photoinduced variation of the surface tension as function of the dye concentration, which

a)Electronic mail: yoshino@ele.eng.osaka-u.ac.jp confirms the possibility of amplification of relatively weak photochemical effect in liquid crystalline media. ${ }^{19}$

Ultrathin, highly ordered freely suspended films play an important role in physics of liquid crystals, providing unique information on the systems of limited dimensionality. ${ }^{20}$ Contrary to the soap bubbles they are very robust and under appropriate conditions, uniformly thick smectic liquid crystal films from many hundreds of layers down to only two molecular layers can be readily prepared and remain stable for weeks. ${ }^{21}$ The principal possibility that thin layers may exist in the form of freely suspended films is related to the presence in smectic phase of internal forces holding of the centers of mass of the molecules within a single layer. When the liquid crystal transforms into nematic phase with chaotic distribution of the centers mass of molecules in the space or isotropic phase, the layered structure disappears and the films lose stability. ${ }^{9}$ Another remarkable property of smectic film, important for the purpose of this letter, is that they, similar to rubber, can be subjected to shear strain or nonhomogeneous displacement in direction of the layer normal. In the limit of small displacement, such smectic films can be considered as elastic membranes in the Rayleigh sense and their transverse vibrations are governed by the two-dimensional wave equation, which, for the case of rectangular film and neglect, any losses have an analytical solution of the form: ${ }^{6}$

$$
\nu=\frac{1}{2} \sqrt{\frac{2 \sigma}{\rho_{s}}\left(\frac{n^{2}}{a^{2}}+\frac{m^{2}}{b^{2}}\right)},
$$

where $a$ and $b$ are, respectively, the length and the width of the film, $\sigma$ is the surface tension, $\rho_{s}=N l \rho_{\mathrm{LC}}+H \rho_{\text {air }}$ is the two-dimensional mass density, $N$ stands for the number of 
smectic layers, $l$ is interlayer distance, $H$ is the thickness of air moving with the film, $\rho_{\mathrm{LC}} l$ and $\rho_{\text {air }}$ are the bulk densities of the liquid crystal and air. The integer $n$ and $m$ denote the normal modes. In practice, the discrete spectrum of pure tones is transformed to a set of separated spectral resonant bands of final width where positions are extremely sensitive to any changes both film parameters and pressure of ambient air. In the present work we change the parameters of the freely suspended film composed of ferroelectric liquid crystal CS-1029 doped with photoactive azobensene dye $D^{*} 55$ by UV and VIS light. The appearance of banana-shaped azobenzene isomers under UV photoexcitation results in the disorganization of the liquid crystal structure accompanying with decrease the spontaneous polarization $P_{s}$ of ferroelectric freely suspended film and surface tension $\sigma$. The chiral dye $D^{*} 55$ was chosen because the UV-light-excited cisisomers in its mixture with smectic crystals show an extraordinary long lifetime. ${ }^{22}$ Therefore, its influence on the film parameters may be measured even after switching the UV light off. As liquid crystal we used the commercial ferroelectric liquid crystal CS-1029, which has the advantage of being in the smectic $C^{*}$ phase at room temperature $\left(\mathrm{Cr}\left[-18^{\circ} \mathrm{C}\right]\right.$ $\mathrm{SmC}^{*} \mathrm{SmA}\left[85^{\circ} \mathrm{C}\right] \mathrm{N}^{*}\left[95^{\circ} \mathrm{C}\right]$ iso). Use of a ferroelectric liquid crystal has a considerably simplified a way of excitation of bending vibration in freely suspended film. ${ }^{2-5}$

\section{RESULTS AND DISCUSSION}

In our experiment, freely suspended smectic $C^{*}$ films were formed by drawing the CS-1029 over a rectangular hole $\left(2 \times 10 \mathrm{~mm}^{2}\right)$ in a glass microscope cover slide. The process of the preparation of the films has been described elsewhere. ${ }^{7}$ The film thickness was measured by standard reflection spectroscopy technique. The films were placed in a thermal jacket $\left( \pm 0.1^{\circ} \mathrm{C}\right)$ equipped with glass window which allowed the simultaneous illumination of the film by both the probing beam of $\mathrm{He}-\mathrm{Ne}$ laser and active light (UV or yellow). Spontaneous polarization $P_{s}$ of CS-1029 was measured by standard triangular voltage method $\left(25 \mathrm{~Hz}, \pm 10 \mathrm{~V}_{\mathrm{p}-\mathrm{p}}\right)$. It should be noted that various classical experimental facilities have been constructed to measure the surface tension of freely suspended films. ${ }^{23-25}$ Here, for measuring of the absolute value of surface tension and its increment we have chosen resonant method ${ }^{2,3,7}$ as the most simple and reliable.

For the present work we first recorded the photochromism spectra of $D^{*} 55$ in dilute chloroform solution Fig. 1(a). The dye has a spectrum typical of azo-compounds with a trans-isomer $\pi$ - $\pi^{*}$ absorption maximum at $\lambda_{m}=350 \mathrm{~nm}$ and an $n-\pi^{*}$ absorbtion maximum at $\lambda_{m}=450 \mathrm{~nm}$. On the irradiation of the solution by UV light, the trans-band completely disappears for about $30 \mathrm{~s}$. The dark relaxation has a characteristic time of about $10 \mathrm{~h}$, and cis-transformation is accelerated dramatically by illumination of the solution with strong yellow light. $P_{s}$ values of CS-1029 were measured by the triangular voltage method $\left(25 \mathrm{~Hz}, \pm 10 \mathrm{~V}_{\mathrm{p}-\mathrm{p}}\right)$. The values of $P_{s}$ of CS-1029 doped with 3 wt $\%$ of $D^{*} 55$ are plotted as a function of the temperature in Fig. 1(b). The magnitude of $P_{s}$ ceased at the $\mathrm{SmC}^{*}-\mathrm{SmA}$ phase transition temperature. Introduction of $3 \%$ azobenzene dye into the liquid crystal

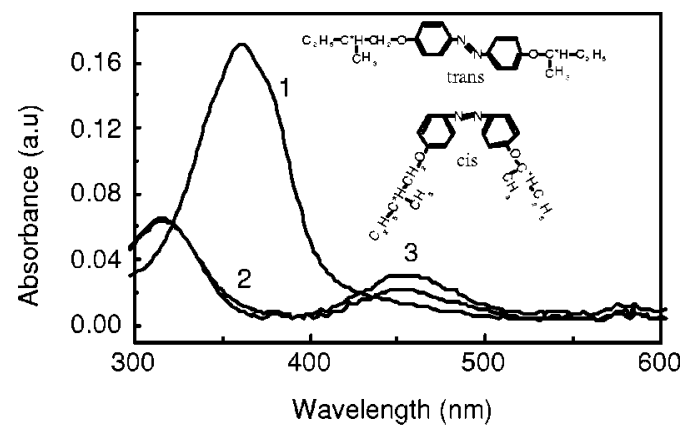

(a)

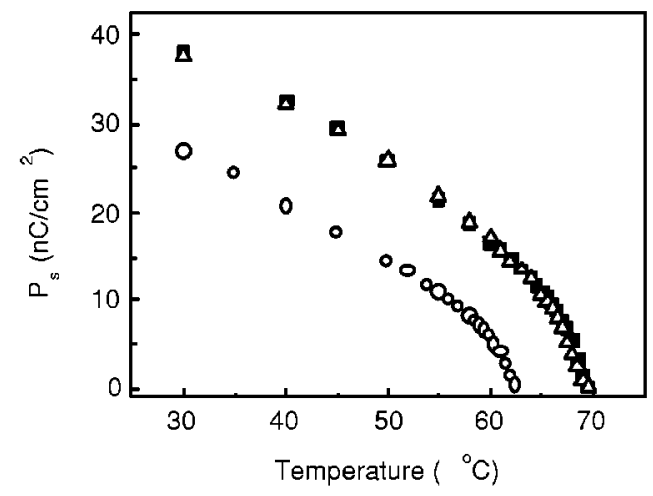

(b)

FIG. 1. Photochemically induced changes in the bulk properties of guesthost systems: (a) Trans-cis process of a $100 \mathrm{mg} / \mathrm{l}$ chloroform solution of $D^{*} 55\left(T=25^{\circ} \mathrm{C}\right.$, filter UVD-35, 300-400 nm, $\left.P=3 \mathrm{~mW} / \mathrm{cm}^{2}\right)$. Upon UV irradiation for $0 \mathrm{~min}$ (curve 1), $30 \mathrm{~s}$ (curve 2), and $6 \mathrm{~min}$ (curve 3); (b) $P_{s}$ of CS-1029 doped with 3 wt $\%$ of chiral azobenzene derivative $D * 55$ as the function of the temperature: $(\boldsymbol{\square})$ before irradiation; $(\bigcirc)$ after irradiation at $360 \mathrm{~nm}(10 \mathrm{~min}) ;(\triangle)$ after irradiation at $450 \mathrm{~nm}(10 \mathrm{~min})$. Inset: chemical structures of trans- and cis-form of chiral dye $D^{*} 55$. In the absence illumination, trans-isomer more stable than cis isomer, but can be converted to cis form by electronic excitation under UV irradiation. The reverse reaction proceeds thermally or on illumination with longer-wavelength light.

phase lowered the $\mathrm{SmC}^{*}-\mathrm{SmA}$ phase transition by $3{ }^{\circ} \mathrm{C}$. After irradiation at $360 \mathrm{~nm}$, the profile changed and phase transition was further lowered by $7{ }^{\circ} \mathrm{C}$ as shown in Fig. 1(b).

For the excitation of transverse film vibrations we exploited linear coupling of lateral electric field with the spontaneous polarization of ferroelectric liquid crystal. ${ }^{2,3,5}$ In this case the azimuthal motion of the liquid crystal director driven by alternating electric field is accompanied by, socalled backflow, which induces a viscous stress disturbing the surface of the film. ${ }^{26}$ The periodical displacement of the film surface results in deflection of the probing beam of lowpower $\mathrm{He}-\mathrm{Ne}$ laser, as shown in the inset of Fig. 2. By passing the beam through an iris diaphragm, the deflection of the beam is converted into an amplitude modulation of laser intensity detected by photodiode. The photodiode response current is analyzed by a lock-in amplifier tuned to the first harmonic of the control voltage.

The typical spectrum of the mechanical bending oscillations of ferroelectric liquid crystal freely suspended film induced by sinusoidal electric field is demonstrated in Fig. 2(a). One can see, that despite the high volume viscosity of CS-1029 $\left(\eta\left[25^{\circ} \mathrm{C}\right]=270 \mathrm{mPas}\right)$ the spectrum displays a 


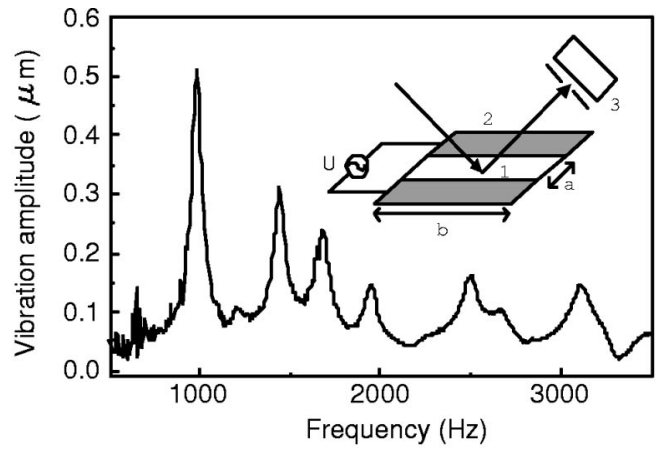

(a)

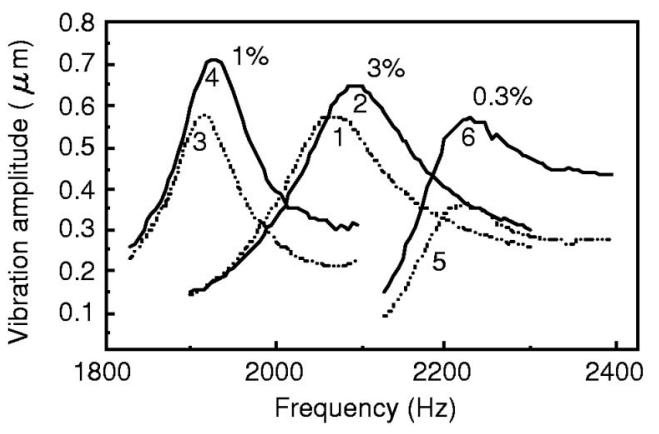

(b)

FIG. 2. Frequency dependence of linear electromechanical effect in ferroelectric freely suspended liquid crystal films: (a) Typical spectrum of electromechanical effect in freely suspended film (film size $2 \times 10 \mathrm{~mm}^{2}$, CS1029 doped with $1 \% D^{*} 55, N=195, T=30.6^{\circ} \mathrm{C}$ ). (b) Shift of spectral bands upon irradiation by UV and yellow light for different concentrations of dye in CS-1029. cis-curves $(1,3,5)$ correspond to illumination of UV (10 min) and trans-curves $(2,4,6)$ are taken upon successive illumination by yellow light (10 min). 1, 2, CS-1029 doped with $3 \%$ wt $D * 55, N=363$, shift of resonance $\Delta \nu=26 \mathrm{~Hz} ; 3,4$, CS-1029 doped with $1 \% D^{*} 55, N=195$, $\Delta \nu=14 \mathrm{~Hz} ; 5,6$, CS-1029 doped with $0.3 \% D * 55, N=255, \Delta \nu=10 \mathrm{~Hz}$. Inset, framework geometry: (1) freely suspended film, (2) electrodes, (3) position sensitive detector.

pronounced resonant character with rather narrow spectral bands $(Q \sim 15-20)$. Actually the narrowness of resonant bands determines sensitivity of the given method to any external influence.

The influence of UV-VIS irradiation on vibration spectra was studied at room temperature. Figure 2(b) shows the UV and yellow light induced changes in vibration amplitudes for three freely suspended films doped with $0.3,1$, and 3 wt $\%$ of $D^{*} 55$. (For convenience we demonstrate the photoinduced changes in the vibration motion of the films by the examples of evolution of appropriate discrete spectral bands.) The spectral curves (1,3, and 5) were recorded after 10 min UV illumination of fresh prepared CS-1029 films with the corresponding concentration of $D^{*} 55$ but the curves $(2,4$, and 6) after the subsequent 10 min illumination of the films with yellow light which returns the system to its initial condition. The UV illumination results both in decrease of the vibrational amplitude and in a considerable shift of the resonance maximum towards lower frequencies. The former effect can be easily explained by decrease in the viscous force moment acting onto the surface of the freely suspended film, due to the decreased $P_{s}$ value in the sample enriched by

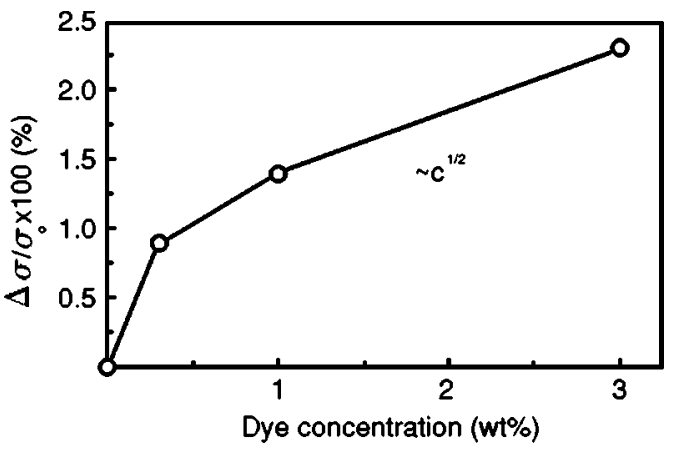

(a)

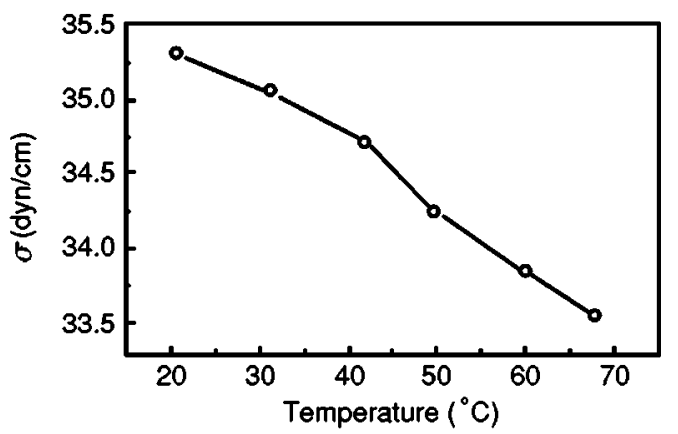

(b)

FIG. 3. Influence of different factors on the surface tension. (a) Concentration dependence of photoinduced variation of the surface tension in the mixtures of CS-1029 doped with $D^{*} 55$; (b) Temperature dependence of surface tension of pure ferroelectric liquid crystal CS-1029.

the cis-form, as follows from Fig. 1(b). On the other hand, the latter effect at isothermal and isobaric conditions is caused by a change in surface tension of the membrane. The photoinduced change in the surface tension, as calculated by Eq. (1), is shown in Fig. 3(a) as a function of dye concentration. The function is clearly nonlinear, in contrast to the similar concentration dependence for mixtures of simple organic liquids which are additive at low concentration of doping molecules. ${ }^{27}$

It can be shown that the frequency shift observed for illuminated films is not caused by film heating. Thus, e.g., the temperature dependence of surface tension value for pure CS-1029 presented in Fig. 3(b) shows that about a $20 \mathrm{~K}$ heating of the freely suspended film is necessary, to produce a $2 \%$ change in the surface tension. Such an overheating should shift guest-host system, e.g., at $50{ }^{\circ} \mathrm{C}$ to the paraelectric SmA phase [see temperature behavior of the curve corresponding to the trans-form, Fig. 1(b) and was not observed]. More evidence is the relaxation of resonant maxima [curves 1, 3, and 5 in Fig. 2(b)] back to their initial positions (curves 2, 4, and 6) during $10 \mathrm{~h}$ after switching off the UV illumination, that corresponding to the characteristic time of dark cis-to-trans relaxation for $D^{*} 55$.

Figure 4 illustrates the evolution of mechanical parameters of the film induced by cis-trans isomerization. The plots present changes in amplitude and phase of film oscillations under illumination with UV and yellow light [Figs. 4(a) and 4(b), respectively]. Data of Figs. 4(a) and 4(b) correspond to 


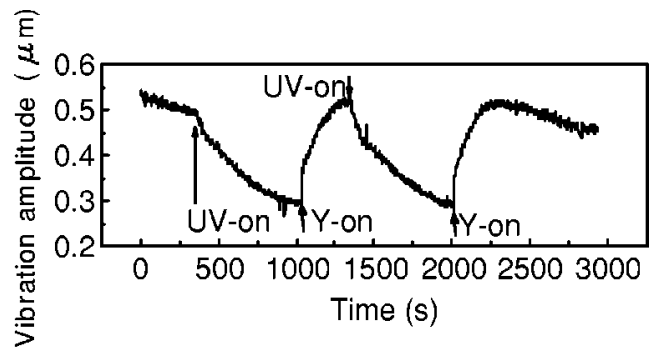

(a)

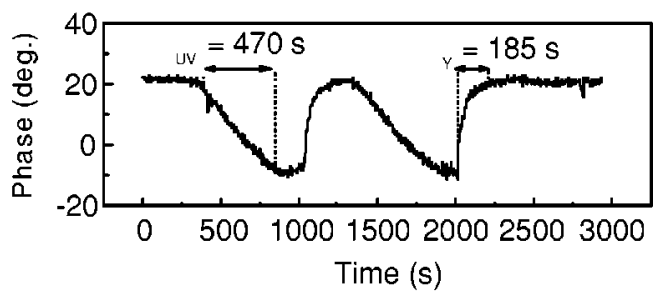

(b)

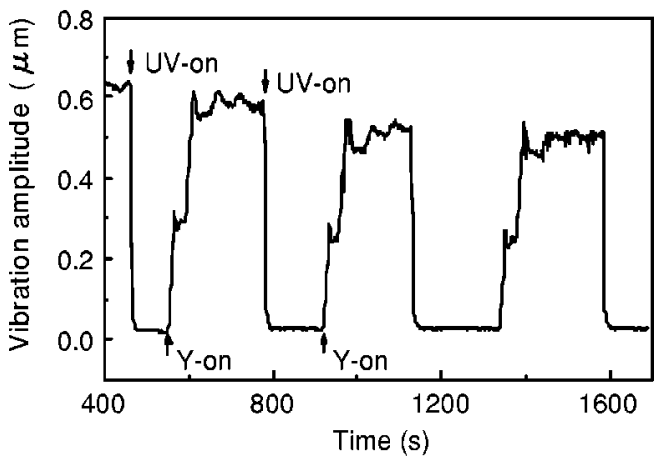

(c)

FIG. 4. Kinetics of the UV and yellow light induced changes in the freely suspended film based on CS-1029 doped with 3 wt \% of $D^{*} 55$ : (a), (b) time dependence of vibration amplitude and phase shift at $T=20.6{ }^{\circ} \mathrm{C}, \tau_{\mathrm{UV}}$ $=470 \mathrm{~s}, \tau_{\mathrm{Y}}=185 \mathrm{~s}$; (c) time dependence of vibration amplitude at $T$ $=65{ }^{\circ} \mathrm{C}, \tau_{\mathrm{UV}}=5 \mathrm{~s}, \tau_{\mathrm{Y}}=60 \mathrm{~s}$ (applied voltage $30 \mathrm{~V}$ on frequency of 2085 $\mathrm{Hz}$, film size $2 \times 10 \mathrm{~mm}^{2}$ ).

a low temperature of $20.6^{\circ} \mathrm{C}$. The relaxation times of cistrans isomerization were measured as $\tau_{\mathrm{UV}}=470 \mathrm{~s}$ and $\tau_{\mathrm{Y}}$ $=185 \mathrm{~s}$ at that temperature but decreased notably when approaching the $\mathrm{SmC}^{*}-\mathrm{SmA}$ transition achieving $\tau_{\mathrm{UV}}=5 \mathrm{~s}$ and $\tau_{\mathrm{Y}}=60 \mathrm{~s}$ at $65^{\circ} \mathrm{C}$. It is particularly remarkable that change in the amplitude of film oscillations at $65^{\circ} \mathrm{C}$ was as high as $100 \%$, as shown in Fig. 4(c). We also notice that at $20.6{ }^{\circ} \mathrm{C}$, the relaxation time for the UV-on process is significantly larger than that for the back relaxation process. This is contrary to the commonly observed behavior ${ }^{22}$ where the former is faster than the latter, as seen in Fig. 4(c) presenting complicated two-step relaxation process at $65^{\circ} \mathrm{C}$. At the moment, such unusual kinetics of UV and yellow light is not clear and certain to be the subject of special investigations.

\section{CONCLUSIONS}

In conclusion, an optomechanical effect has been observed and described in freely suspended films of liquid crystals. The reported effect can be used in numerous technical applications including as, e.g., remote adjustment of the mechanical characteristics of the elastic membrane by UV and VIS light. On the other hand, the effect highlights the fundamental problems of condensed matter physics related to the cooperative phenomena of the soft matter in the twodimensional systems.

${ }^{1}$ S. Uto, E. Tazoh, M. Ozaki, and K. Yoshino, J. Appl. Phys. 82, 2791 (1997).

${ }^{2}$ S. V. Yablonskii, T. Oue, H. Nambu, A. S. Mikhailov, M. Ozaki, and K. Yoshino, Appl. Phys. Lett. 75, 64 (1999).

${ }^{3}$ S. V. Yablonskii, A. S. Mikhailov, K. Nakano, M. Ozaki, and K. Yoshino, JETP 93, 94 (2001).

${ }^{4}$ E. I. Demikhov, S. A. Pikin, and E. S. Pikina, Phys. Rev. E 52, 6250 (1995).

${ }^{5}$ S. Uto, M. Ozaki, and K. Yoshino, Appl. Phys. Lett. 74, 117 (1999).

${ }^{6}$ P. M. Morse, Vibration and Sound (McGraw-Hill, New York, 1936).

${ }^{7}$ P. Pieranski, L. Beliard, J.-Ph. Tournellec, X. Leoncini, C. Furtlehner, H. Dumoulin, E. Riou, B. Jouvin, J.-P. Fenerol, Ph. Palaric, J. Heuving, B. Cartier, and I. Kraus, Physica A 194, 364 (1993).

${ }^{8}$ I. Kraus, Ch. Bahr, I. V. Chikina, and P. Pieranski, Phys. Rev. E 58, 610 (1998).

${ }^{9}$ E. I. Kats and V. V. Lebedev, Fluctuational Effects in the Dynamics of Liquid Crystals and Film (Springer, New York, 1993).

${ }^{10} \mathrm{~S}$. V. Yablonskii, K. Nakano, S. A. Mikhailov, M. Ozaki, and K. Yoshino, Appl. Phys. Lett. 80, 571 (2002).

${ }^{11}$ T. Ikeda, M. Nakano, Y. Yu, O. Tsutsumi, and A. Kanazawa, Adv. Mater. (Weinheim, Ger.) 15, 201 (2003).

${ }^{12}$ H. Finkelmann and E. Nishikawa, Phys. Rev. Lett. 87, 015501 (2001).

${ }^{13}$ T. A. Krentsel, O. D. Lavrentovich, and S. Kumar, Mol. Cryst. Liq. Cryst. 320, 77 (1998).

${ }^{14}$ W. R. Folks, Yu. A. Reznikov, S. N. Yarmolenko, and O. D. Lavrentovich, Mol. Cryst. Liq. Cryst. 292, 183 (1997).

${ }^{15}$ T. Ikeda, T. Sasaki, and K. Ichimura, Nature (London) 361, 428 (1993).

${ }^{16}$ T. Sasaki, T. Ikeda, and K. Ichimura, J. Am. Chem. Soc. 116, 625 (1994).

${ }^{17}$ T. Sasaki and T. Ikeda, J. Phys. Chem. 99, 13002 (1995).

${ }^{18}$ T. Sasaki and T. Ikeda, J. Phys. Chem. 99, 13013 (1995).

${ }^{19}$ Y. Lansac, M. A. Glaser, N. A. Clark, and O. D. Lavrentovich, Nature (London) 398, 54 (1999).

${ }^{20}$ R. Pindak and D. Moncton, Phys. Today 5, 57 (1982).

${ }^{21}$ T. Stoebe, P. Mach, and C. C. Huang, Phys. Rev. E 49, R3587 (1994).

${ }^{22}$ L. M. Blinov, M. V. Kozlovski, T. Nagata, M. Ozaki, and K. Yoshino, Jpn. J. Appl. Phys., Part 2 38, L1042 (1999).

${ }^{23}$ R. Stannarius and C. Cramer, Liq. Cryst. 23, 371 (1997).

${ }^{24}$ P. Mach, C. C. Huang, and H. T. Nguyen, Phys. Rev. Lett. 80, 732 (1998).

${ }^{25}$ A. Boudaoud, Y. Couder, and M. B. Amar, Phys. Rev. Lett. 82, 3847 (1999).

${ }^{26}$ A. Jakli and N. Eber, in Modern Topics in Liquid Crystals: Piezoelectric Effects in Liquid Crystals, edited by A. Buka (World Scientific, New York, 1993).

${ }^{27}$ J. Escobedo and G. A. Mansoori, AIChE J. 44, 2324 (1998). 